\title{
Exploring the receptor origin of vibration-induced reflexes
}

\author{
Mustafa A. Yildirim ${ }^{1} \cdot$ Betilay Topkara ${ }^{2} \cdot$ Tugba Aydin $^{1} \cdot$ Nurdan Paker $^{1} \cdot$ Derya Soy $^{1} \cdot$ Evrim Coskun $^{1}$. \\ Kadriye Ones ${ }^{1} \cdot$ Aysenur Bardak $^{1} \cdot$ Nur Kesiktas $^{1} \cdot$ Mustafa G. Ozyurt $^{2} \cdot$ Berna Celik $^{1} \cdot$ Burcu Onder $^{1} \cdot$ Aysegul Kılıc $^{1}$. \\ Habib C. Kucuk $\mathbb{D}^{1} \cdot$ Ilhan Karacan $\mathbb{D}^{1} \cdot$ Kemal S. Türker ${ }^{2}$
}

Received: 3 October 2019 / Revised: 7 January 2020 / Accepted: 8 January 2020 / Published online: 15 January 2020

(c) The Author(s), under exclusive licence to International Spinal Cord Society 2020

\begin{abstract}
Study design An experimental design.

Objectives The aim of this study was to determine the latencies of vibration-induced reflexes in individuals with and without spinal cord injury (SCI), and to compare these latencies to identify differences in reflex circuitries.

Setting A tertiary rehabilitation center in Istanbul.

Methods Seventeen individuals with chronic SCI (SCI group) and 23 participants without SCI (Control group) were included in this study. Latency of tonic vibration reflex (TVR) and whole-body vibration-induced muscular reflex (WBVIMR) of the left soleus muscle was tested for estimating the reflex origins. The local tendon vibration was applied at six different vibration frequencies $(50,85,140,185,235$, and $265 \mathrm{~Hz})$, each lasting for $15 \mathrm{~s}$ with 3 -s rest intervals. The WBV was applied at six different vibration frequencies $(35,37,39,41,43$, and $45 \mathrm{~Hz})$, each lasting for $15 \mathrm{~s}$ with 3-s rest intervals. Results Mean (SD) TVR latency was 39.7 (5.3) $\mathrm{ms}$ in the SCI group and 35.9 (2.7) ms in the Control group with a mean (95\% CI) difference of -3.8 (-6.7 to -0.9 ) ms. Mean (SD) WBV-IMR latency was 45.8 (7.4) $\mathrm{ms}$ in the SCI group and 43.3 (3.0) $\mathrm{ms}$ in the Control group with a mean $(95 \% \mathrm{CI})$ difference of -2.5 ( -6.5 to 1.4$) \mathrm{ms}$. There were significant differences between TVR latency and WBV-IMR latency in both the groups (mean (95\% CI) difference; $-6.2(-9.3$ to -3.0$) \mathrm{ms}, p=$ 0.0001 for the SCI group and $-7.4(-9.3$ to -5.6$) \mathrm{ms}, p=0.011$ for Control group).
\end{abstract}

Conclusions The results suggest that the receptor of origin of TVR and WBV-IMR may be different.

\section{Introduction}

Whole-body vibration (WBV) is a mode of exercise training that is increasingly used in sports training and neurological rehabilitation because some believe it improves the neuromuscular system [1,2]. Although there is as yet no clear neurophysiological explanation for improvements, these may be due to the tonic vibration reflex (TVR). TVR, a polysynaptic motor response, is considered to result from

Supplementary information The online version of this article (https:// doi.org/10.1038/s41393-020-0419-5) contains supplementary material, which is available to authorized users.

Ilhan Karacan

ilhankaracan@hotmail.com

1 İstanbul Physical Medicine Rehabilitation Training and Research Hospital, Istanbul, Turkey

2 Physiology Department, Koç University School of Medicine, Istanbul, Turkey the vibration-induced activity of the muscle spindle [1-3]. Despite these claims usually from anecdotal evidence, there has been no direct evidence on the receptor origin of TVR. Since the pathway of the reflex induced by WBV is not identified, the spinal reflex induced by WBV is referred to as the WBV-induced muscular reflex (WBV-IMR) in the literature $[4,5]$.

Although one of the main characteristics of reflex pathway is its latency defined as the time between activation of the receptor (e.g., muscle spindle) and muscle activation [4], there is no previous study reporting the latency of TVR due to the absence of an accepted method to estimate it. There are two major reasons for this methodological shortage to evaluate the TVR latency; first, an interval between two consecutive vibration stimulus is shorter than even the shortest reflex latency; second, the vibratory stimulus is a continuous event, therefore, there is no easy way to determine the exact temporal location of the effective stimulation. These two obstacles have recently been overcome by using a technique called the cumulative average method [5]. 
Using the cumulative average method, our previous study has shown that WBV induces short- and long-latency reflexes depending on the vibration amplitude $[5,6]$. The short-latency WBV-IMR elicited during low amplitude $(0.23 \mathrm{~mm})$ vibration is likely to have a muscle spindle origin as its latency $(34 \mathrm{~ms})$ was similar to the tendon reflex (T-reflex) latency. Long-latency WBV-IMR, on the other hand, occurs during a medium $(1.25 \mathrm{~mm})$ and high $(2.20 \mathrm{~mm})$ amplitude vibration. Due to its longer latency (44 ms) compared with the T-reflex, its pathway has been suggested to be different from the pathway of the T-reflex [6]. Briefly, despite some signs that the receptor origin of the WBV-IMR may be muscle spindle, it has not yet been clearly identified.

Evaluation of muscle spindle-based reflex responses in patients with spasticity may shed light on the receptor origin of the WBV-IMR and TVR. Stretch reflex and tendon jerks are muscle spindle-based reflex responses [7]. These reflex responses and the muscle tone are increased in patients with spasticity [8]. In addition to the change in amplitude of the spindle-based reflex responses, reflex latency may also change due to activation of autophagy in the dorsal root ganglia, demyelination, and trans-synaptic degeneration in people with SCI [9-15]. Therefore, we expect that the reflex latencies of people with spinal cord injury (SCI) may be different compared with able-bodied individuals. If the latencies of both TVR and WBV-IMR are not equally affected by the neuropathological processes associated with $\mathrm{SCI}$, it can be considered that the receptor origin of WBVIMR is different from the receptor origin of TVR proposed as a muscle spindle-based reflex.

Reflex latency are determined by using electromyography $[4,5]$. H-reflex and T-reflex are the analogues of stretch reflex which can be detected by electromyography $[3,6,7]$. To determine whether the receptor origin of the TVR and WBV-IMR is muscle spindle, we compared the latency of TVR and WBV-IMR with the latency of the T-reflex. Furthermore, we wished to determine the reflex latencies in both individuals with and without SCI so that we could compare and contrast results. We propose the following hypotheses:

(1) The latency of TVR claimed to be a polysynaptic spindle reflex is longer than the latency of T-reflex a monosynaptic spindle reflex.

(2) The latency of TVR is similar to the latency of WBVIMR triggered by the high amplitude WBV.

(3) The latencies of H-reflex, T-reflex, TVR, and WBVIMR are equally affected in individuals with SCI.

If the above hypotheses are confirmed, this study provides evidence supporting the view that the receptor origin of TVR and WBV-IMR is muscle spindle and that the beneficial neuromuscular effects of WBV can be explained by TVR.

\section{Methods}

This study is an experimental study. The unique protocol IDs were IstPMRTRH-BMR1 and NCT03424551 at ClinicalTrials.gov ID.

\section{Participants}

Twenty-six individuals with chronic SCI and 27 ablebodied participants were screened for eligibility. The inclusion and exclusion criteria are provided in the Supplementary file. Ultimately, 17 people with SCI (SCI group) and 23 able-bodied individuals (Control group) met the inclusion criteria and were included in the study. Volunteers who were not suitable for electrophysiological tests (reflex latency measurement) and WBV or TVR were excluded from the study. Nine individuals with SCI and four ablebodied participants were excluded from the study due to reasons reported in the Supplementary file.

The characteristics for each participant with SCI are given in Table 1. The mean (standard deviation, SD) age of the participants with and without SCI was 39.5 years (10.5) and 27.7 years (7.0), respectively, and their corresponding mean (SD) heights were $174.4 \mathrm{~cm}(11.2)$ and $175.0 \mathrm{~cm}$ (8.4), respectively. There were 2 females and 15 males in the SCI group, and 8 females and 15 males in the Control group, respectively. The mean time since injury of participants in the SCI group was 45.4 months and ranged from 1 to 198 months. Eight individuals had a complete SCI (AIS A) and nine had an incomplete SCI (AIS B, C, or D). The neurologic level was C5-7 in five and T3-L3 in twelve of the individuals in the SCI group.

Ethical approval was obtained from the University of Health Sciences Sadi Konuk Training \& Research Hospital Ethics Committee (Approval Number, 2017-08-14) and a written informed consent was obtained from each subject prior to the experimental procedures.

\section{Testing procedures}

T-reflex, H-reflex, TVR, and WBV-IMR of the left soleus muscle were tested in both groups of participants.

\section{Data recording}

Surface EMG (SEMG), all accelerometers, stimulator, and electronic reflex hammer data were obtained using a data acquisition system (POWERLAB ${ }^{\circledR}$ ADInstruments Co, Oxford, United Kingdom). The sampling rate was $10 \mathrm{kHz}$ 
Table 1 Characteristics for each participant with SCI.

\begin{tabular}{lllllll}
\hline Case & Age $(\mathrm{yrs})$ & Gender & Neurological level & AIS Scale & SCI duration (months) & Medication \\
\hline AA & 32 & Male & T11 & A & 58 & Baclofen \\
AY & 55 & Male & T12 & B & 1 & Baclofen \\
BM & 35 & Male & C7 & B & 198 & Baclofen \\
BP & 42 & Male & C5 & D & 16 & Baclofen \\
CC & 36 & Male & T11 & A & 13 & Pregabalin \\
EA & 39 & Male & T12 & A & 108 & Pregabalin \\
HB & 51 & Male & T11 & D & 17 & Baclofen \\
HK & 57 & Female & L1 & D & 3 & Baclofen \\
HK & 20 & Male & T4 & A & 5 & Baclofen \\
MA & 38 & Male & C5 & D & 121 & Baclofen \\
MO & 33 & Male & T3 & A & 135 & Baclofen \\
OE & 37 & Male & C7 & B & 53 & Pregabalin \\
SC & 44 & Male & T12 & D & 7 & Baclofen \\
SK & 54 & Male & T11 & B & 9 & Baclofen \\
SNK & 22 & Female & T11 & A & 3 & Pregabalin \\
TE & 42 & Male & C5 & A & 6 & Baclofen \\
TY & 35 & Male & L3 & A & 19 &
\end{tabular}

for all channels. All records were taken whilst quietly seated with the knees and hips at a $90^{\circ}$ angle. Surface EMG was recorded from the left soleus muscle using a bipolar recording configuration. The reference electrode was placed on the medial malleolus. The electrodes were placed on the soleus muscle according to the SENIAM guidelines [16]. Prior to electrode placement, the skin was shaved, lightly abraded, and cleaned with alcohol wipes. Self-adhesive, disposable, $\mathrm{Ag}-\mathrm{AgCl}$ disc electrodes with a disc radius of $10 \mathrm{~mm}$ (KENDALL ${ }^{\circledR}$ Coviden; Massachusetts, USA) were used. The electrode cables were fixed to the body to prevent the electrodes from swaying.

The recorded data were processed and analyzed offline using LabChart7 ${ }^{\circledR}$ Software Version V7.3.3 (PowerLab $^{\circledR}$ system ADInstruments, Oxford, UK).

\section{Determination of T-reflex latency}

An electronic reflex hammer (Elcon, Germany) was used to obtain T-reflex response.

The left Achilles tendon was tapped twenty times with $\sim 5 \mathrm{~s}$ intervals between consecutive taps. Since the T-reflex was taken at rest, their SEMG recordings were filtered using a bandpass filter with the cut-off frequency of 5 and $500 \mathrm{~Hz}$. The onset of the first deflection on the tap stimulus trace (determined visually) was marked as a trigger point to average the rectified EMG traces between the time of the trigger and $75 \mathrm{~ms}$ segment after the trigger. The standard error (SE) values for each data point on the averaged EMG records were calculated. The lowest SE on the averaged of the EMG data was considered as the onset of T-reflex spike (Fig. 1).

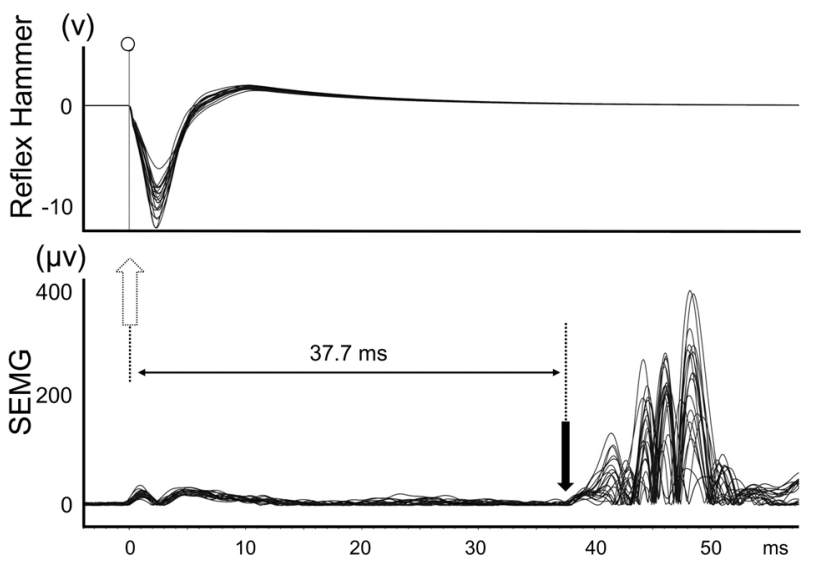

Fig. 1 T-reflex latency measurement. Representative T-reflex latency values of one male individual with SCI (EA). The open circle and arrow show the onset of the tap stimulus that was used as the trigger to average the rectified EMG data. The solid arrow represents the onset of the EMG spike.

\section{Determination of H-reflex latency}

A cathode $(5 \times 5 \mathrm{~mm})$ was placed at the midpoint of the left popliteal fossa and an anode $(10 \times 10 \mathrm{~cm})$ was placed immediately proximal to the patella to evoke the H-reflex for monopolar stimulation of the tibial nerve [17]. A monophasic electrical current was delivered as square pulses with a width of $1 \mathrm{~ms}$ using a stimulator (FE155 Stimulator HC ADInstrument, Oxford, UK). Interstimulus interval was $\sim 5 \mathrm{~s}$. Since the H-reflex was taken at rest, their SEMG recordings were filtered using a bandpass filter from 5 to $500 \mathrm{~Hz}$. 


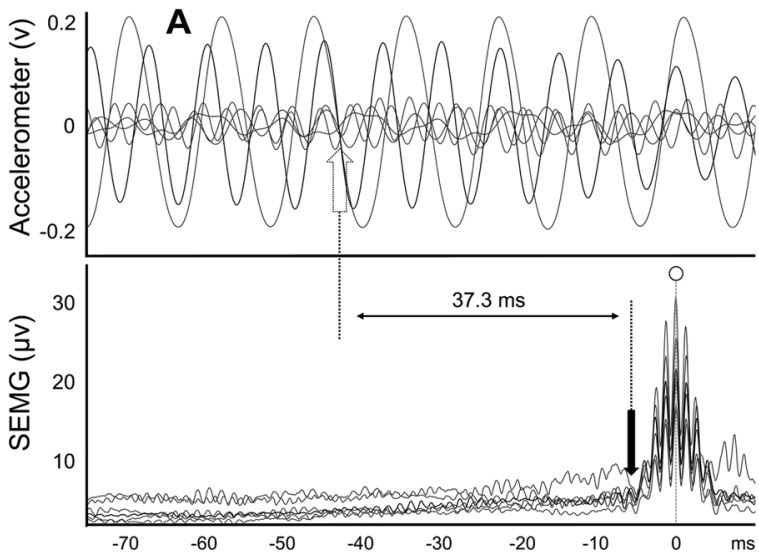

Fig. 2 Reflex latency measurement using cumulated average method. a TVR latency measurement and, b WBV-IMR latency measurement. Representative TVR latency values of one male individual with SCI (EA). The open circle represents the positive peak of

To determine the H-reflex latency, onset of electrical stimulus marked automatically by an algorithm of the LabChart program was used as triggers to average the rectified EMG traces between the time of the trigger and 75 $\mathrm{ms}$ segment after the trigger. The lowest SE on the averaged of the EMG data was considered as the onset of an $\mathrm{H}$ reflex spike.

\section{Determination of TVR latency}

The local vibration was applied to the left Achilles tendon by using a custom-made vibrator. An accelerometer (LIS344ALH; Ecopack, Mansfield, TX, USA) was fixed to the head of vibrator (vibrator accelerometer) to determine TVR latency. Six different vibration frequencies $(50,85$, $140,185,235$, and $265 \mathrm{~Hz}$ ), each lasting for $15 \mathrm{~s}$ with $3-\mathrm{s}$ rest intervals were delivered in random order to negate any order effect. Approximately $3 \mathrm{~kg}$ of force was applied to maintain the vibrator head in contact with the skin during vibration. Accelerometer recordings were filtered with a high-pass filter set at $5 \mathrm{~Hz}$. SEMG recordings were filtered using a bandpass filter from 270 to $500 \mathrm{~Hz}$ in order to reduce the vibratory waveforms in the SEMG [18]. TVR latency was determined by using the cumulative average method [5]. Latency was expressed as milliseconds (ms) (Fig. 2).

\section{Determination of WBV-IMR latency}

The WBV was applied by using Power Plate ${ }^{\circledR}$ Pro5 (PowerPlate International, Amsterdam, The Netherlands). The participants were barefoot and their left leg placed on the WBV platform. No sponge or foam was placed between the vibration platform and the foot. The right foot of each

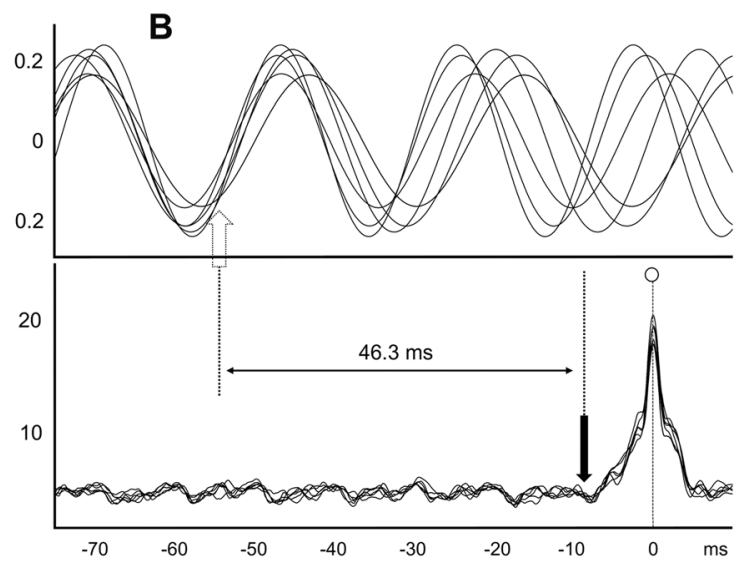

the rectified EMG that was used as the trigger to average the accelerometer data and the rectified EMG data. The solid arrow represents the onset of the EMG spike, and the open arrow represents the stimulus onset point.

participant was placed on an isolated platform. A high amplitude $(2.2 \mathrm{~mm})$ vibration was applied.

An accelerometer (LIS344ALH; Ecopack, Mansfield, TX, USA) was fixed to WBV platform (platform accelerometer) to determine WBV-IMR latency. Six different vibration frequencies $(35,37,39,41,43$, and $45 \mathrm{~Hz})$, each lasting for $15 \mathrm{~s}$ with 3 -s rest intervals were delivered in random order to negate any order/time effect. Accelerometer recordings were filtered with a high-pass filter set at $5 \mathrm{~Hz}$. SEMG recordings were filtered using a bandpass filter from 80 to $500 \mathrm{~Hz}$ [18]. WBV-IMR latency was calculated by using the cumulative average method [5] (Fig. 2). The cumulative average method is a new technique for the estimation of the latency of reflexes induced by the high rate stimulation of the neuromuscular system, such as WBV and tendon vibration stimulation [5]. Since a vibration cycle generates a reflex response on the EMG at a fixed latency, it is possible to calculate reflex latency using a simple averaging method. To achieve this goal, the peak of the EMG response was used as the trigger and sinusoidal acceleration record as the source in a spike-triggered averaging algorithm. This procedure was performed for all six vibration frequencies that were tested. The averaged data belonging to different vibration frequencies were then superimposed to generate cumulated average. SE for the cumulated average records was then obtained. The lowest value of the SE indicated timing of the effective stimulus (Fig. 2). This averaging procedure also indicated the initiation of the EMG response to the effective stimulus. Therefore, the reflex latency for such a complex stimulus as vibration became very clear; from the point of effective stimulus to the point of initial EMG response.

All latencies were adjusted to the body height of each participant. 


\section{Statistical analyses}

All data were checked for normal distribution using the Shapiro-Wilk test. Arithmetic mean and SD were calculated for each variable. The comparisons of the normalized latencies were performed using the Wilcoxon test. The comparisons of the normalized latencies between groups were calculated using Mann-Whitney $U$-test or unpaired $t$-test, depending on suitability of data with normal distribution. A $p$ value less than 0.05 was considered as significant. The software package used for data management was PASW Statistic 18. Post Hoc Power analysis was performed using G*Power software (version 3.1.9.2, Franz Paul, Universität Kiel, Germany). To obtain information regarding the magnitude of between-group differences, nonparametric effect sizes were calculated based on the $z$-values obtained from the Mann-Whitney $U$-tests (effect size $=z / \sqrt{ } n$ ). Effect sizes were categorized as follows: small effect $=0.1$; medium effect $=$ 0.3 ; and large effect $=0.5$ [19].

\section{The power analysis of this study}

The present study found a significant difference between TVR latency and WBV-IMR latency. The effect size was 0.925 for the SCI group and 1.776 for the Control group. Running a power analysis, the computed power was 0.947 for the SCI group and 1.00 for the Control group.

\section{Results}

The mean (SD) latency of TVR was 39.7 (5.3) $\mathrm{ms}$ in the SCI group and 35.9 (2.7) $\mathrm{ms}$ in the Control group with a mean $(95 \% \mathrm{CI})$ difference of $-3.8(-6.7$ to -0.9$) \mathrm{ms}$. The mean (SD) WBV-IMR latency was 45.8 (7.4) $\mathrm{ms}$ in the SCI group and 43.3 (3.0) $\mathrm{ms}$ in the Control group with a mean (95\% CI) difference of -2.5 ( -6.5 to 1.4 ) $\mathrm{ms}$. There was no significant difference between TVR latency and T-reflex latency in either the SCI or Control group ( $p=0.926, p=$ 0.999 , respectively). There were however significant differences between TVR latency and H-reflex latency in both the SCI and Control groups $(p=0.0001, p=0.0001$, respectively). Furthermore, there was a significant difference between TVR latency and WBV-IMR latency in both the SCI and Control groups $(p=0.011, p=0.0001$, respectively). There was also a significant difference between T-reflex latency and WBV-IMR latency in both the SCI and Control groups $(p=0.003, p=0.0001$, respectively) (Table 2, Fig. 3).

When we compared the latency differences between the SCI and Control groups, the latency of H-reflex $(p=0.006)$, T-reflex $(p=0.011)$, and TVR $(p=0.013)$ were significantly longer in the SCI group while there was no
Table 2 Adjusted latencies of reflex response (milliseconds).

\begin{tabular}{lclll}
\hline & H-reflex & T-reflex & TVR & WBV-IMR \\
\hline $\begin{array}{l}\text { Individuals } \\
\text { with SCI }\end{array}$ & $34.0(3.4)$ & $38.6(3.4)$ & $39.7(5.3)$ & $45.8(7.4)$ \\
Control group & $31.2(1.6)$ & $36.2(1.9)$ & $35.9(2.7)$ & $43.3(3.0)$ \\
$P$ value & $0.006^{\mathrm{a}}$ & $0.011^{\mathrm{a}}$ & $0.013^{\mathrm{a}}$ & $0.194^{\mathrm{b}}$ \\
Effect size & 0.39 & 0.40 & 0.43 & 0.44 \\
\hline
\end{tabular}

Data were given as arithmetic mean (SD)

${ }^{a}$ Mann-Whitey $U$-test

${ }^{\mathrm{b}}$ Unpaired $t$-test

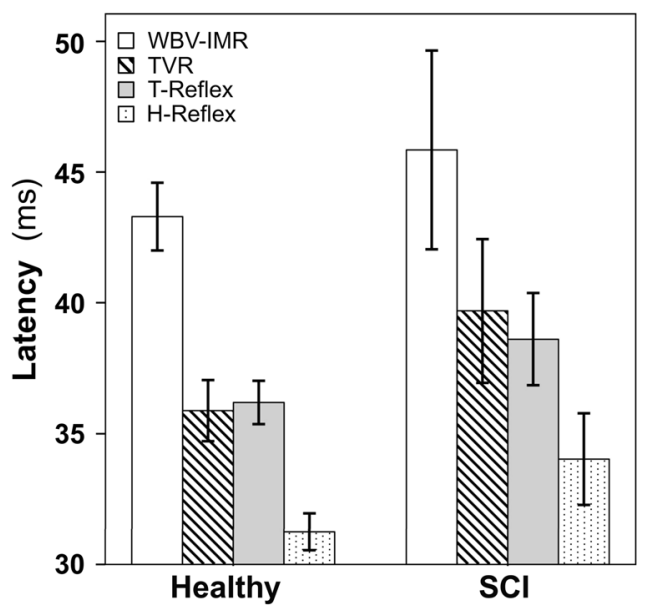

Fig. 3 Adjusted latencies of the various reflex responses for the able-bodied participants and individuals with SCI. Error bars with $95 \%$ confidence interval of the reflex latencies.

difference in WBV-IMR latency between the two groups $(p=0.194)$ (Table 2).

\section{Discussion}

It is widely believed that the beneficial effects of WBV on the neuro-musculoskeletal system occur via a spinal reflex such as TVR [1, 2]. This study has discovered three original findings indicating that WBV activates a reflex pathway other than TVR. Firstly, we have for the first time estimated the reflex latency of TVR using a recognized method, namely the cumulated average method [5]. In contrast to our first hypothesis we have discovered that the TVR latency matches the latency for the T-reflex. Secondly, in contrast to our second hypothesis, we have also discovered that the TVR latency is significantly different from the WBV-IMR. Thirdly, we have shown that the latency of all spindle-based reflexes including TVR are higher in the SCI group than Control group, whilst WBV-IMR latency is similar in both populations. In other words, the latency of both TVR and WBV-IMR are not equally affected by the neuropathological 
process associated with SCI which is contradictory to our third hypothesis.

\section{Estimation of the TVR latency}

Quoniam et al. claimed to have measured TVR latency in their studies. They measured the latency as an interval between the onset of vibration and onset of an increase in baseline EMG activity and, estimated the latency of the TVR as $450 \mathrm{~ms}$ [20]. However, for any nervous activity to be called "reflex", it needs to initiate before the minimum reaction time for that stimulus [21]. Furthermore, the time between the onset of vibration and onset of an increase in baseline EMG activity was reported to vary considerably with the vibration amplitude [22]. The problem of vibrationinduced reflex latency measurement has now been overcome by the use of the cumulative average method [5]. Thus, using this novel method we found the latency for the TVR to be about $35-40 \mathrm{~ms}$.

The cumulative average method is a new technique for the estimation of the latency of reflexes induced by the high frequency stimulation of the neuromuscular system, such as vibration stimuli [5]. Since a vibration cycle generates a reflex response on the EMG at a fixed latency, it is possible to calculate reflex latency using a simple averaging method. To achieve this goal, the peak of the EMG response is used as the trigger and sinusoidal acceleration record as the source in a spike-triggered averaging algorithm. This procedure is done for all six vibration frequencies that were tested. All the averaged data belonging to different vibration frequencies are then superimposed to generate cumulated average. SE for the cumulated average records is also obtained. The lowest value of the SE indicates timing of the effective stimulus (Fig. 2). This averaging procedure also indicates the initiation of the EMG response to the effective stimulus. Therefore, the reflex latency for such a complex stimulus as vibration become very clear; from the point of effective stimulus to the point of initial EMG response.

\section{TVR latency versus other "spindle-based" reflexes and WBV-IMR}

In contrast to our first hypothesis, we have discovered that the TVR latency matches the latency for the T-reflex. On the other hand, we found that TVR latency was longer than the H-reflex latency. H-reflex, analogous to TVR and Treflex, is obtained by stimulating Ia afferents electrically bypassing the muscle spindle [23]. The difference between the TVR and H-reflex latencies can be explained by bypassing muscle spindle in the H-reflex studies. The latency difference between TVR and H-reflex was found to be similar to the latency difference between T-reflex and H-reflex (Table 2).
Contrary to our second hypothesis, the present study showed that the TVR latency was significantly shorter than the WBV-IMR latency. This result indicates that the receptor origin and/or afferent of WBV-IMR are different from those of TVR.

\section{Reflex latencies in both groups}

Another interesting finding of the present study is that while the latencies of all spindle-based reflexes (e.g., H-reflex, Treflex and TVR) were longer in the SCI group than in the Control group, WBV-IMR latencies of individuals in the SCI and Control group were similar. This finding refutes our third hypothesis which suggested that TVR latency and WBV-IMR latency are affected equally by SCI. This finding also supports the above idea that the pathways of TVR and WBV-IMR may be different.

There may be several pathological processes that affect reflex latencies after SCI. When the spinal cord is injured, the primary injury caused by the initial traumatic event are followed by the secondary injuries created by a series of biological and functional changes including activation of autophagy in the dorsal root ganglia, demyelination, and trans-synaptic degeneration [9-12]. Demyelination following SCI is a result of rapid death of oligodendrocytes which are vulnerable to damage. Because of this vulnerability, oligodendrocytes at considerable distances from the lesion can also undergo apoptosis. Demyelination begins in the acute phase (up to 2 days) of SCI and is a chronic progressive feature of SCI. Large myelinated sensory neurons may be preferentially affected by pathology. After demyelination, myelinated sheath reappears around the axons gradually in the chronic phase of injury. However, thickness and length of this regenerated myelin are smaller than in intact axons, leading to a decrease in conduction velocity and an increase in the reflex latency [9-11].

A high risk of axonal loss of peripheral nerves is reported due to activation of autophagy in the dorsal root ganglia or trans-synaptic degeneration in individuals with SCI in previous studies. Trans-synaptic degeneration occurs when lower motor neurons are disconnected from upper motor neurons, which causes dysfunction or loss of lower motor neurons. Under normal circumstances, the upper motor neuron exerts a trophic effect on the amount of protein synthesis (e.g., antifibrillation factor) in the lower motor neuron, such that after an upper motor neuron lesion less protein would be synthesized [12-15]. Kirshblum et al. reported that a statistically significant reduction in nerve conduction velocity was found in the lower limbs of people chronic complete tetraplegia compared with able-bodied participants. They attributed the decrease in nerve conduction velocity to trans-synaptic degeneration [14]. 
We hypothesized that TVR latency and WBV-IMR latency were equally affected by the changes in the motor neuron pool activity or secondary injury processes such as axonal degeneration and demyelination in individuals with SCI. However, our findings do not confirm this hypothesis. The controversy between our hypothesis and these results can be explained with the help of trans-synaptic degeneration. It may be assumed that WBV-IMR affects predominantly slow-twitch muscle fibers and TVR affects predominantly fast-twitch muscle fibers. The basis of this assumption is built on the knowledge that the motor axons innervating slow-twitch muscle units are thinner and their conduction velocities are slower than the motor axons innervating fast-twitch muscle units [24].

Supporting this assumption, $\mathrm{Li}$ et al. found that spinal cord transection causes decreased axonal transport in the thick motor axons of the high-threshold motor units but not in the thinner motor axons of the low-threshold motor units [25]. Similarly, Lukács et al. reported that the selective loss of high-threshold motor units following the upper motor neuron lesion is due to trans-synaptic degeneration [26]. Following trans-synaptic degeneration and the consequent muscle denervation, the muscle fibers can be reinnervated through collateral nerve sprouts originating from the intact motor neurons, thereby causing an enlargement of the remaining motor units during the chronic stage of SCI [27]. However, after loss of high-threshold motor units and its thick myelinated motor axons, the thinner myelinated motor axons with the slower conduction velocity, remains. Transsynaptic degeneration therefore can explain our longer latency findings in individuals with SCI.

Aging is another important factor affecting the latency of stretch reflexes which are muscle spindle-based reflexes $[6,28,29]$. The latency of the soleus stretch reflex has been reported to be longer in the elderly than young people [30]. Furthermore, aging predominantly results in atrophy of myelinated primary sensory neurons. There exists a preferential loss in anatomical structure and physiological function of large myelinated fibers with aging and the involvement of sensory fibers occurs earlier than motor fibers. A diverse and nonuniform decline of sensory structures and physiological function occurs across the life span [31]. Regarding SCI, Siegenthaler et al. stated that there is an age-associated increase in demyelination, accompanied by an age-associated progressive decrease in remyelination following SCI [11]. Since the mean age of the individuals in the SCI group was significantly higher than the Control group in the present study, it can be assumed that age contributed to the observed differences in the latency between the two groups.

The limitation of this study was that the required sample size could not be calculated before the experiments as there was no similar study investigating the TVR latency.
However, post hoc power analysis showed that the power of this study was reliably high.

In conclusion, for the first time, this study estimated TVR latency and showed that TVR latency was almost identical to T-reflex latency suggesting that the receptor of origin of the TVR is likely to be the muscle spindles. Since there were significant differences between TVR latencies and WBV-IMR latencies between the groups we suggest that the neuronal pathways of TVR and WBV-IMR may be different. WBV is increasingly used in clinical or sportive rehabilitation due to its beneficial effects on the neuromuscular system. This study indicates that WBV activates a reflex pathway other than TVR. A better understanding of the mechanisms underlying receptor origin will help form scientific basis for WBV to be used more effectively and efficiently in rehabilitation. Further studies are needed to identify the precise afferent type and/or receptor origin of WBV-IMR pathway and muscle fiber type predominantly affected by TVR or WBV.

\section{Data availability}

The datasets generated and/or analyzed during the current study are available from the corresponding author on reasonable request.

Acknowledgements We thank all persons who participated in this study.

Funding This research received no specific grant from any funding agency in the public, commercial, or not-for-profit sectors.

Author contributions All authors were responsible for study conception and design, and drafting of manuscript. MAY, TA, NP, DS, EC, $\mathrm{KO}, \mathrm{AB}, \mathrm{NK}, \mathrm{BC}, \mathrm{BO}, \mathrm{AK}$, and $\mathrm{HCK}$ were also involved in data acquisition. IK, KST, MGO, and BT were also involved in statistical analysis and data interpretation. IK and KST were additionally responsible for project management, data interpretation, and finalizing the manuscript.

\section{Compliance with ethical standards}

Conflict of interest The authors declare that they have no conflict of interest.

Ethical statement We certify that all applicable institutional and governmental regulations concerning the ethical use of human volunteers were followed during the course of this research.

Publisher's note Springer Nature remains neutral with regard to jurisdictional claims in published maps and institutional affiliations.

\section{References}

1. Alam MM, Khan AA, Farooq M. Effect of whole-body vibration on neuromuscular performance: a literature review. Work. 2018; 59:571-83. 
2. Liao LR, Pang MYC. Effect of whole-body vibration on neuromuscular activation of leg muscles during dynamic exercises in individuals with stroke. J Strength Cond Res. 2017;31:1954-62.

3. De Gail P, Lance JW, Neilson PD. Differential effects on tonic and phasic reflex mechanisms produced by vibration of muscles in man. J Neurol Neurosurg Psychiatry. 1966;29:1-11.

4. Türker KS. Reflex control of human jaw muscles. Crit Rev Oral Biol Med. 2002;13:85-104.

5. Karacan I, Cakar H, Sebik O, Yılmaz G, Cidem M, Kara S, et al. A new method to determine reflex latency induced by high rate stimulation of the nervous system. Front Hum Neurosci. 2014;8:536. https://doi.org/10.3389/fnhum.2014.00536.

6. Karacan I, Cidem M, Cidem M, Türker KS. Whole-body vibration induces distinct reflex patterns in human soleus muscle. J Electromyogr Kinesiol. 2017;34:93-101.

7. Matthews PB. The reflex excitation of the soleus muscle of the decerebrate cat caused by vibration applied to its tendon. J Physiol. 1966;184:450-72.

8. Sheean G. Neurophysiology of spasticity. In: Barnes MP, Johnson GR, editors. Upper motor neurone syndrome and spasticity Clinical management and neurophysiology. Cambridge University Press, New york, 2008. p. 9-64.

9. Totoiu MO, Keirstead HS. Spinal cord injury is accompanied by chronic progressive demyelination. J Comp Neurol. 2005;486 (4):373-83.

10. Hassannejad Z, Yousefifard M, Azizi Y, Zadegan SA, Sajadi K, Sharif-Alhoseini M, et al. Axonal degeneration and demyelination following traumatic spinal cord injury: a systematic review and meta-analysis. J Chem Neuroanat. 2019;97:9-22.

11. Siegenthaler MM, Ammon DL, Keirstead HS. Myelin pathogenesis and functional deficits following SCI are age-associated. Exp Neurol. 2008;213:363-71.

12. Hou H, Zhang L, Zhang L, Liu D, Xiong Q, Du H, et al. Acute spinal cord injury could cause activation of autophagy in dorsal root ganglia. Spinal Cord. 2013;51:679-82.

13. Zheng C, Zhu Y, Lu F, Ma X, Tian D, Jiang J. Trans-synaptic degeneration of motoneurons distal to chronic cervical spinal cord compression in cervical spondylotic myelopathy. Int J Neurosci. 2017;127:988-95.

14. Kirshblum S, Lim S, Garstang S, Millis S. Electrodiagnostic changes of the lower limbs in subjects with chronic complete cervical spinal cord injury. Arch Phys Med Rehabil. 2001;82: 604-7.

15. Van De Meent H, Hosman AJ, Hendriks J, Zwarts M, EM-SCI Study Group, Schubert M. Severe degeneration of peripheral motor axons after spinal cord injury: a European multicenter study in 345 patients. Neurorehabil Neural Repair. 2010;24:657-65.
16. Hermens HJ, Freriks B, Disselhorst-Klug C, Rau G. Development of recommendations for SEMG sensors and sensor placement procedures. J Electromyogr Kinesiol. 2000;10:361-74.

17. Özyurt MG, Shabsog M, Dursun M, Türker KS. Optimal location for eliciting the tibial H-reflex and motor response. Muscle Nerve. 2018;58:828-33.

18. Sebik O, Karacan I, Cidem M, Türker KS. Rectification of SEMG as a tool to demonstrate synchronous motor unit activity during vibration. J Electromyogr Kinesiol. 2013;23:275-84.

19. Fritz CO, Morris PE, Richler JJ. Effect size estimates: current use, calculations, and interpretation. J Exp Psychol Gen. 2012;141: 2-18.

20. Quoniam C, Hay L, Roll JP, Harlay F. Age effects on reflex and postural responses to propriomuscular inputs generated by tendon vibration. J Gerontol A Biol Sci Med Sci. 1995;50:B155-65.

21. Brodin P, Miles TS, Türker KS. Simple reaction-time responses to mechanical and electrical stimuli in human masseter muscle. Arch Oral Biol. 1993;38:221-6.

22. Desmedt JE, Godaux E. Vibration-induced discharge patterns of single motor units in the masseter muscle in man. J Physiol. 1975;253:429-42.

23. Palmieri RM, Ingersoll CD, Hoffman MA. The hoffmann reflex: methodologic considerations and applications for use in sports medicine and athletic training research. J Athl Train. 2004;39: 268-77.

24. Nordlander RH. Motoneurons of the tail of young Xenopus tadpoles. J Comp Neurol. 1986;253:403-13.

25. Li JY, Kling-Petersen A, Dahlström A. Influence of spinal cord transection on the presence and axonal transport of CGRP-, chromogranin A-, VIP-, synapsin I-, and synaptophysin-like immunoreactivities in rat motor nerve. J Neurobiol. 1992;23: 1094-110.

26. Lukács M, Vécsei L, Beniczky S. Large motor units are selectively affected following a stroke. Clin Neurophysiol. 2008;119: 2555-8.

27. Hara Y, Masakado Y, Chino N. The physiological functional loss of single thenar motor units in the stroke patients: when does it occur? Does it progress? Clin Neurophysiol. 2004;115:97-103.

28. Mynark RG, Koceja DM. Effects of age on the spinal stretch reflex. J Appl Biomech. 2001;17:188-203.

29. Peddireddy A, Wang K, Svensson P, Arendt-Nielsen L. Influence of age and gender on the jaw-stretch and blink reflexes. Exp Brain Res. 2006;171:530-40.

30. Obata H, Kawashima N, Akai M, Nakazawa K, Ohtsuki T. Agerelated changes of the stretch reflex excitability in human ankle muscles. J Electromyogr Kinesiol. 2010;20:55-60.

31. Shaffer SW, Harrison AL. Aging of the somatosensory system: a translational perspective. Phys Ther. 2007;87:198-207. 